\title{
ARTICLE OPEN \\ Biaxial strain tuning of the optical properties of single-layer transition metal dichalcogenides
}

Riccardo Frisenda ${ }^{1}$, Matthias Drüppel ${ }^{2}$, Robert Schmidt ${ }^{3}$, Steffen Michaelis de Vasconcellos ${ }^{3}$, David Perez de Lara ${ }^{1}$, Rudolf Bratschitsch ${ }^{3}$, Michael Rohlfing ${ }^{2}$ and Andres Castellanos-Gomez ${ }^{1,4}$

Since their discovery, single-layer semiconducting transition metal dichalcogenides have attracted much attention, thanks to their outstanding optical and mechanical properties. Strain engineering in these two-dimensional materials aims to tune their bandgap energy and to modify their optoelectronic properties by the application of external strain. In this paper, we demonstrate that biaxial strain, both tensile and compressive, can be applied and released in a timescale of a few seconds in a reproducible way on transition metal dichalcogenides monolayers deposited on polymeric substrates. We can control the amount of biaxial strain applied by letting the substrate expand or compress. To do this, we change the substrate temperature and choose materials with a large thermal expansion coefficient. After the investigation of the substrate-dependent strain transfer, we performed microdifferential spectroscopy of four transition metal dichalcogenides monolayers $\left(\mathrm{MoS}_{2}, \mathrm{MoSe}_{2}, \mathrm{WS}_{2}, \mathrm{WSe} \mathrm{C}_{2}\right)$ under the application of biaxial strain and measured their optical properties. For tensile strain, we observe a redshift of the bandgap that reaches a value as large as $95 \mathrm{meV} / \%$ in the case of single-layer $\mathrm{WS}_{2}$ deposited on polypropylene. The observed bandgap shifts as a function of substrate extension/compression follow the order $\mathrm{MoSe}_{2}<\mathrm{MoS}_{2}<\mathrm{WSe}_{2}<\mathrm{WS}_{2}$. Theoretical calculations of these four materials under biaxial strain predict the same trend for the material-dependent rates of the shift and reproduce well the features observed in the measured reflectance spectra.

npj 2D Materials and Applications (2017)1:10; doi:10.1038/s41699-017-0013-7

\section{INTRODUCTION}

Strain engineering has been proposed as a prospective route to modify the electronic and optical properties of two-dimensional (2D) materials. ${ }^{1-4}$ The interest in this topic is motivated by their resilience to mechanical deformations. These systems stand deformations of the order of $10 \%, 5,6$ while conventional 3D semiconductors break at quite moderate deformations of $0.5-1.5 \%{ }^{7}$ Apart from the mechanical toughness, another key question of strain engineering of 2D materials is how they can be conveniently and reproducibly strained. While 3D systems are typically stressed by epitaxially growing them onto substrates with a certain lattice parameter mismatch, strain in 2D systems can be applied more directly by folding, ${ }^{8}$ stretching ${ }^{9-14}$ and bending. ${ }^{15-25}$ Moreover, strain in 2D materials can be easily varied continuously in time, helping to achieve a modulation of the electronic properties. Experiments on $\mathrm{MoS}_{2}$ single-layer and few-layer flakes have already demonstrated that the optical band gap is tunable by $50 \mathrm{meV} / \%$ for uniaxial $\operatorname{strain}^{15,16}$ and $100 \mathrm{meV} / \%$ for biaxial strain. ${ }^{14}$ These results open the door to fabricate devices whose optical and electronic properties can be externally controlled by the application of strain. ${ }^{26}$

Most of the strain engineering experiments to date have been mainly focused on uniaxial strain under static conditions. However, time-dependent straining is desirable for many applications, such as sensors, optical modulators or active optic devices. Here, we explore the use of biaxial strain to modulate the reflectivity of single layer transition metal dichalcogenides (TMDCs) on a timescale of a few seconds. We investigate the strain transfer from thermally expanded or compressed polydimethylsiloxane (PDMS) substrate to a $\mathrm{MoS}_{2}$ monolayer. PDMS is a polymer commonly used in strain engineering experiments with 2D materials. However, we find that this substrate is not efficient in compressing or extending the $2 \mathrm{D}$ material on top. Switching to polypropylene (PP) substrates we find that biaxial strain can be applied reproducibly without slippage up to a maximum tensile biaxial strain of $1 \%$. We investigate the effects of strain on the optical properties of single-layers $\mathrm{MoS}_{2}, \mathrm{MoSe}_{2}, \mathrm{WS}_{2}$ and $\mathrm{WSe}_{2}$. To our knowledge, this is the first experimental investigation of $\mathrm{MoSe}_{2}, \mathrm{WS}_{2}$ and $\mathrm{WSe}_{2}$ under the application of biaxial strain. For increasing tensile strain, a redshift of the optical band gap of these 2D TMDCs is observed, which reaches, in the case of $\mathrm{WS}_{2}$, the large value of $95 \mathrm{meV}$ for a substrate strain of only $1 \%$. The observed bandgap shifts as a function of substrate extension/compression follow the order $\mathrm{MoSe}_{2}<\mathrm{MoS}_{2}<\mathrm{WSe}_{2}<$ $\mathrm{WS}_{2}$, i.e., with $\mathrm{WS}_{2}$ providing the largest bandgap tunability and $\mathrm{MoSe}_{2}$ the lowest. Using the thermal expansion mismatch between a $2 \mathrm{D}$ material and a substrate is a simple but powerful way to achieve biaxial expansion or compression of the 2D material, which is technically more difficult to achieve than uniaxial strain. This method can be readily applied to other $2 \mathrm{D}$ materials and be used to vary the strain in real time.

\footnotetext{
${ }^{1}$ Instituto Madrileño de Estudios Avanzados en Nanociencia (IMDEA-nanociencia), Campus de CantoblancoE-28049 Madrid, Spain; ${ }^{2}$ Institute for Solid-state Theory, University of Münster, D-48149 Münster, Germany and ${ }^{3}$ Institute of Physics and Center for Nanotechnology, University of Münster, D-48149 Münster, Germany

Correspondence: Riccardo Frisenda (riccardo.frisenda@imdea.org) or Michael Rohlfing (michael.rohlfing@uni-muenster.de) or Andres Castellanos-Gomez (andres.castellanos@csic.es)

${ }^{4}$ Present address: Instituto de Ciencia de Materiales de Madrid (ICMM-CSIC), Campus de Cantoblanco, E-28049, Madrid, Spain
}

Received: 23 November 2016 Revised: 1 February 2017 Accepted: 24 February 2017

Published online: 25 May 2017 
In order to apply biaxial strain to single-layer TMDCs, we changed the temperature of the substrate with a Peltier heater/ cooler and exploit the large mismatch between the thermal expansion coefficients of the substrate and the TMDC flake deposited on top, similarly to previous work by part of the authors. ${ }^{12}$ In this work, however, it was not possible to directly determine the substrate expansion and the biaxial strain was limited only to tensile strain. The selected substrate could not effectively transfer the strain to the 2D layer because of its low Young's modulus and only static strain was studied. ${ }^{11,27}$ Here, we present a simple method to accurately calibrate the substrate expansion and we extend the straining method also to compressive strain. By analysing the nature of the strain transfer mechanism, we find a substrate that optimises it and we study time-dependent strain.

\section{RESULTS}

Figure 1a shows a sketch of the thermal expansion calibration method. In the calibration procedure, we measure the distance between periodical features, patterned on the surface of the polymeric substrate by recording optical images of the substrate while changing the temperature with a Peltier element $\left(10-110^{\circ} \mathrm{C}\right)$. Figure $1 \mathrm{~b}$ displays two fragments of optical images of a PDMS substrate with periodic holes (diameter $3 \mu \mathrm{m}$ and pitch $4 \mu \mathrm{m}$ ) taken at a temperature of $30^{\circ} \mathrm{C}$ and $110^{\circ} \mathrm{C}$. Thanks to the presence of the array of holes the thermal expansion of the PDMS substrate is readily visualised when comparing the two images.

We analyse the spacing between holes at different temperatures by studying the autocorrelation function of the intensity of the images, which provides a powerful tool to extract periodical features as explained in Supplementary Information Section S1. For each temperature $T$, we extract the distance $L(T)$ between adjacent holes. The change in distance between holes $\Delta L$ is

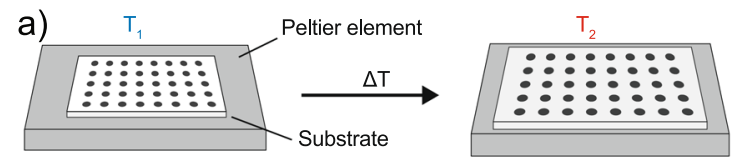

b)

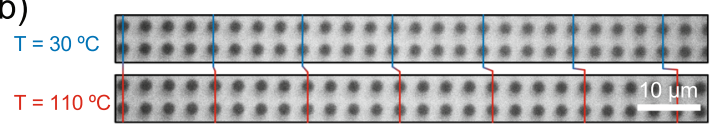

c)

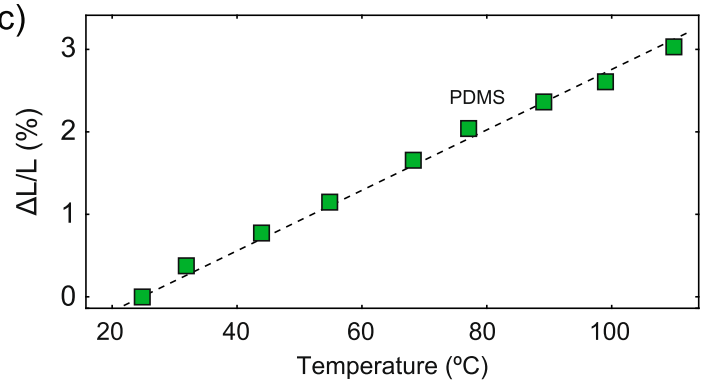

Fig. 1 a Schematic of the temperature-dependent experiment. Substrate heating (cooling) causes the thermal expansion (contraction) of the substrate that induces biaxial strain on the flake predeposited on top. Control over the temperature is achieved with a Peltier heating/cooling element. b Optical microscope image (in grey scale) of a PDMS substrate with periodic holes taken at two different temperatures ( 30 and $110^{\circ} \mathrm{C}$ ). Notice the expanded substrate at higher temperature. c Average percentage increase of the distance between holes as a function of temperature. The dashed line is a linear fit to the data. The slope yields the expansion coefficient of PDMS related to the change in temperature $T$ according to:

$$
\frac{L(T)-L\left(T=25^{\circ} \mathrm{C}\right)}{L\left(T=25^{\circ} \mathrm{C}\right)}=\frac{\Delta L}{L}=a_{\mathrm{sub}} \cdot\left(T-25^{\circ} \mathrm{C}\right),
$$

where $a_{\text {Sub }}$ is the thermal expansion coefficient of the material. Figure 1c shows the percentage change in distance as a function of the temperature of the PDMS substrate. The observed dependence is linear and the linear thermal expansion coefficient can be directly extracted from this measurement, yielding a value $a_{\text {PDMS }}=(3.4 \pm 0.3) \cdot 10^{-4}{ }^{\circ} \mathrm{C}^{-1}$, in good agreement with the value reported in literature. ${ }^{28}$ One can easily determine the amount of biaxial expansion/compression $\varepsilon_{\text {Sub }}$ of the substrate directly from the temperature $\varepsilon_{\text {sub }}=a_{\text {sub }}\left(\mathrm{T}-25^{\circ} \mathrm{C}\right)$. The thermal expansion of this substrate is roughly 50 times larger than that of TMDCs. ${ }^{5}, 29$ Therefore, a change in temperature of the substrate is expected to yield a biaxial strain of the TMDCs deposited on the substrate.

We first studied the effect of strain on mechanically exfoliated flakes of monolayer $\mathrm{MoS}_{2}$ deposited on PDMS, a substrate that is commonly used in strain engineering experiments with 2D materials. The flakes have been deposited onto the PDMS substrates by mechanical exfoliation with Nitto SPV 224 tape. Subsequent deterministic placement ${ }^{30}$ allows one to transfer the flakes from the PDMS to PP and PC substrates. The thickness of the flakes is determined by a combination of quantitative optical analysis, differential reflectance spectroscopy and Raman spectroscopy. Figure $2 \mathrm{a}$ shows an optical image of a flake of $\mathrm{MoS}_{2}$ deposited on a PDMS substrate with different numbers of layers of $\mathrm{MoS}_{2}$ showing a different optical contrast. Figure $2 \mathrm{~b}$ displays the Raman signal, measured at the point indicated in Fig. 1a, which shows two prominent maxima, which are well fitted to Lorentzian functions and attributed to $A_{1 g}$ and $E_{2 g}^{1}$ vibrations of $\operatorname{MoS}_{2}{ }^{31,} 32$ We find a frequency difference $\Delta f=(19.6 \pm 0.2) \mathrm{cm}^{-1}$ between these vibrations, which confirms that the dark region in the optical image is a monolayer of $\mathrm{MoS}_{2}$, since it is known that $\Delta f$ increases with the number of layers as shown in the inset of Fig. $2 \mathrm{~b}$.

To study the optical properties of the monolayer as a function of biaxial strain, we use a micro-reflectance setup in which we illuminate a small area (diameter $\approx 60 \mu \mathrm{m}$ ) of the sample with a halogen white light source, which is collimated through a small diaphragm and focused with the microscope lens (see Supplementary Information Fig. SI5). Thanks to a fibre optic (core diameter $105 \mu \mathrm{m}$ ) attached to the trinocular, we collect only the light reflected by a small area of the sample (diameter $2.1 \mu \mathrm{m}$, see Supplementary Information Fig. S16). The dimensions of this probed area are much smaller than a typical single-layer region that measures at least $10 \times 10 \mu \mathrm{m}$. The differential reflectance signal of an ultra-thin film adsorbed on a surface is directly proportional to the absorbance of the film, ${ }^{33,} 34$ see Supplementary Information section 3 . Figure $2 \mathrm{c}$ shows differential reflectance spectra acquired on the single-layer region of the $\mathrm{MoS}_{2}$ flakes. The differential reflectance spectra of single-layer $\mathrm{MoS}_{2}$ increase for energies larger than the bandgap (optical band gap: $1.9 \mathrm{eV}$ ). On top of a broad background, one finds two prominent maxima centred at 1.90 and $2.05 \mathrm{eV}$, which correspond to the A and B excitons. Photons are absorbed due to the direct transitions at the $\mathrm{K}$ point of the Brillouin zone of monolayer $\mathrm{MoS}_{2}{ }^{35-38}$

The differential reflectance spectra have been recorded at room temperature $\left(25^{\circ} \mathrm{C}\right)$ and at $55^{\circ} \mathrm{C}$. This temperature difference causes an expansion of the substrate of approximately $1 \%$. An inspection of the spectra reveals that both the $A$ and $B$ resonances display a redshift of $13 \mathrm{meV}$ for this substrate expansion. To extract the energy of the excitons from Fig. 2c, we fit the peaks present in the differential reflectance spectra with Lorentzian functions. In Fig. 2d, we display the energy shift of the A and B excitons, with respect to the room temperature values, as a function of the thermal expansion of the substrate. The data indicate a linear dependence on the substrate expansion both for 

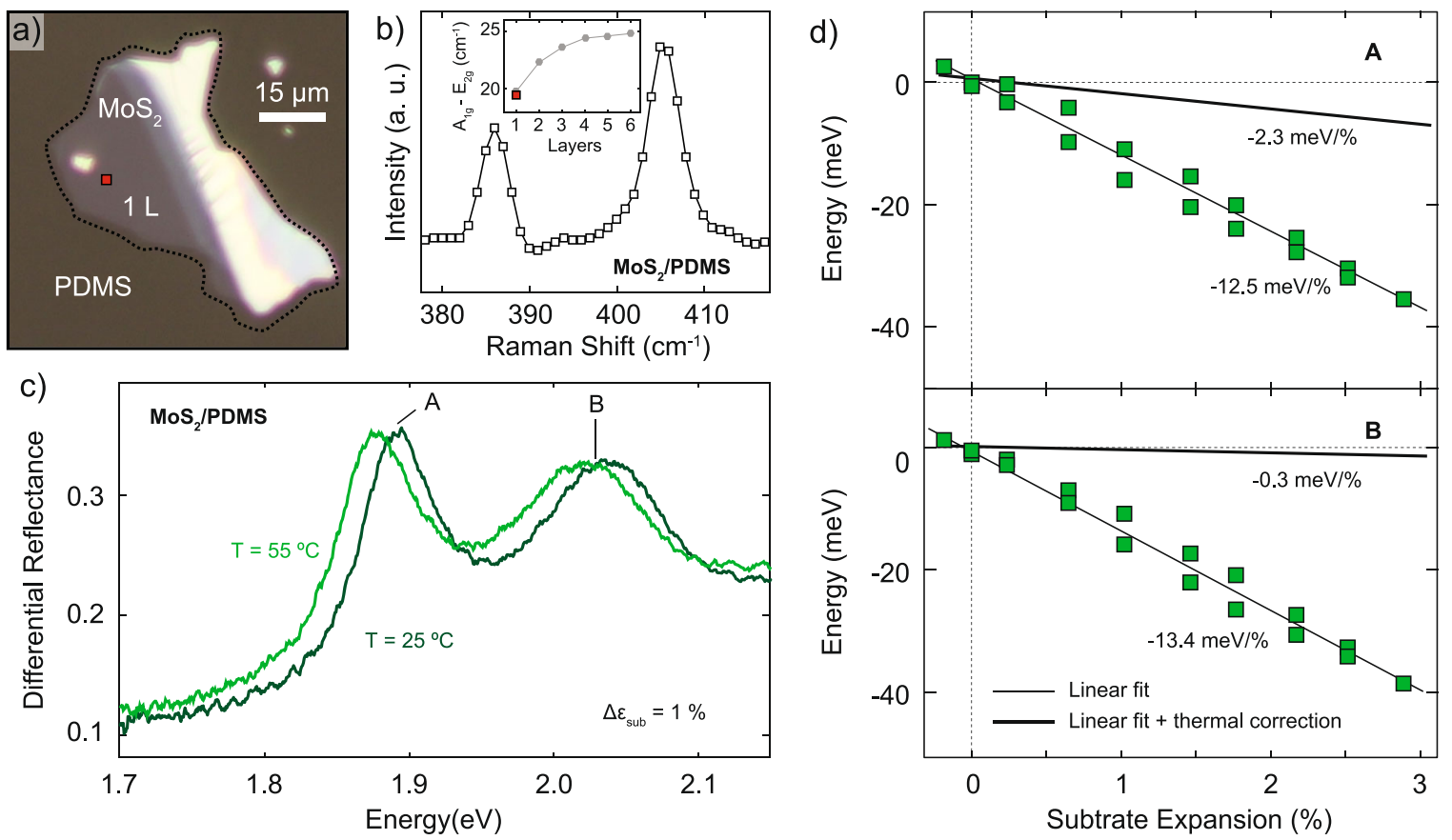

Fig. 2 a Optical image of a $\mathrm{MoS}_{2}$ flake deposited on a PDMS substrate; the darker violet region is single-layer MoS 2 . The contour of the flake is outlined by a black dashed line. b Raman spectrum of the $\mathrm{MoS}_{2}$ flake taken at the position of the red square in panel (a). The inset shows the dependence of the difference in Raman shift of the modes $\mathrm{A}_{1 \mathrm{~g}}$ and $\mathrm{E}_{2 \mathrm{~g}}$ as a function of the number of layers, the red square indicates the value found in this study. c Differential reflectance measured on the single-layer region of the $\mathrm{MoS}_{2}$ flake for zero substrate expansion (light coloured curve) and at $1 \%$ of substrate expansion (dark curve). d Energy of the excitonic peaks A, B in panel (c), extracted from a multi-peak fit as a function of the substrate expansion. The thin lines represent a linear fit to the data, while the thick line represents the same trend after subtraction of the intrinsic thermal dependence contribution

Table 1. Experimental gauge factors of single-layer $\mathrm{MoS}_{2}$

\begin{tabular}{lll}
\hline Exciton & MoS2/PDMS (meV/\%) & MoS2/PP (meV/\%) \\
\hline A & -12.5 & -51.1 \\
& $(-2.2)$ & $(-25.4)$ \\
B & -13.4 & -48.7 \\
& $(-1.0)$ & $(-17.8)$ \\
\hline
\end{tabular}

Gauge factor for excitons $\mathrm{A}$ and $\mathrm{B}$ of $\mathrm{MoS}_{2}$ single-layer flakes deposited on PDMS and PP substrates extracted from applying biaxial strain on the different $2 D$ flakes. The numbers between brackets are the gauge factor with the thermal component removed

tensile and for compressive strain. We find a gauge factor, i.e. the rate of shift in energy of a spectral feature as a function of the substrate percentage expansion/compression, of approximately $-13 \mathrm{meV} / \%$ (corresponding to $-0.44 \mathrm{meV} /{ }^{\circ} \mathrm{C}$, see Table 1 ). To test the reproducibility and rule out slippage, we studied the differential reflectance of single-layer $\mathrm{MoS}_{2}$ during consecutive cycles of warming/cooling of the PDMS substrate (see Supplementary Information Fig. SI16, additional measurements on polycarbonate are discussed in the in Fig. SI12).

To investigate the intrinsic effect of the temperature on the optical spectrum of a single layer $\mathrm{MoS}_{2}$ flake, we repeated the warming/cooling experiment using glass as a substrate. Glass has a small thermal expansion coefficient $\left(a_{\text {Glass }}=0.04 \cdot 10^{-4}{ }^{\circ} \mathrm{C}^{-1}\right)$, which is approximately two orders of magnitude smaller than the coefficients of polymeric substrates, resulting in an expected maximum strain attainable of only $0.04 \%$ when heating from 10 to $110^{\circ} \mathrm{C}$. Supplementary Information Fig. SI11 shows the differential reflectance spectra of single-layer $\mathrm{MoS}_{2}$ deposited on glass for different temperatures. The $A$ and $B$ excitons redshift for increasing temperature. From a linear fit, we find the intrinsic thermal dependence of the energy of the $A$ and $B$ excitonic peak of monolayer $\mathrm{MoS}_{2}$ equal to -0.34 and $-0.42 \mathrm{meV} /{ }^{\circ} \mathrm{C}$, respectively. These values are in good agreement with previous measurements of $\mathrm{MoS}_{2}$ on $\mathrm{SiO}_{2}$ and of single-layer $\mathrm{MoSe}_{2}$ on $\mathrm{SiO}_{2}{ }^{35,}{ }^{39}$ and are attributed to the redshift of the bandgap energy caused by the thermal expansion of the lattices of these single-layer TMDCs. After subtraction of this intrinsic thermal shift from the measured gauge factor of $\mathrm{MoS}_{2}$ on PDMS, the energy shift of the excitons induced by straining the polymer substrate is between 0 and -2 $\mathrm{meV} / \%$. In contrast, our ab-initio calculations of the absorption spectra of $\mathrm{MoS}_{2}$ upon biaxial strain show a much more pronounced shift. We perform density functional theory (DFT) calculations in the local density approximations (LDAs), followed by a GW step within the LDA + GdW approximation, ${ }^{40}$ to then solve the Bethe-Salpeter equation (BSE) to access absorption spectra. Figure 3 displays our results for a single-layer $\mathrm{MoS}_{2}$ in absence of external strain and with $1 \%$ of biaxial tensile and compressive strain. We find a linear dependence of both the $A$ and $B$ excitons. The gauge factors for the quasiparticle gap and the $A$ and $B$ excitons are compared in Table 2. An extended table including the quasiparticle gaps and energetic positions of the $A$ and $B$ excitons can be found in the Supplementary Information (Table SI2). The difference between the calculated gauge factors and those measured using PDMS substrates is a factor of 100 smaller. The fact that the redshift observed for $\mathrm{MoS}_{2} / \mathrm{PDMS}$ is comparable in magnitude to the one observed for $\mathrm{MoS}_{2} /$ glass, even if the strain of PDMS is a factor 50 larger than the strain attainable in glass, together with the large discrepancy between the theoretical and the experimental gauge factors indicate that 
most of the strain present in the PDMS substrate is not transferred to the adsorbed $\mathrm{MoS}_{2}$ flake.

According to previous studies on graphene, ${ }^{27}$ the efficiency of a substrate to transfer strain to a flake depends on the Young's modulus of the substrate $E_{\text {Sub }}$. The expected maximum strain $\varepsilon$ induced in the monolayer TMDCs flakes is typically a fraction of the substrate strain $\varepsilon_{\text {Sub }}$ and can be written as:

$\varepsilon=g \cdot \varepsilon_{\text {sub }}$,

with $g$ being a dimensionless parameter that depends on $E_{\text {Sub }}$ and on the lateral size of the monolayer, and has values between 0 and $1 .{ }^{11}$ This is supported by finite element simulations (shown in Supplementary Information Figs. SI17 and SI18) in which we perform an axisymmetric simulation of a $\mathrm{MoS}_{2}$ flake (diameter 10 $\mu \mathrm{m}$, height $0.7 \mathrm{~nm}$ ) on top of a substrate (diameter $500 \mu \mathrm{m}$, height $100 \mu \mathrm{m})$. We calculate the amount of strain transferred from the expanded substrate to the $\operatorname{MoS}_{2}$ flake $\left(E_{M o S 2} \approx 350 \mathrm{GPa}^{11}\right)$ as a function of the substrate's Young's modulus and we find for substrates with a Young's modulus comparable to that of PDMS $\left(E_{\text {Sub }} \approx 500 \mathrm{kPa}\right.$ ) that the strain transferred is on the order of $1 \%$ $(g=0.01)$. To transfer a larger amount of strain, one should use substrates with Young's moduli larger than $500 \mathrm{MPa}$. Among the different possible materials, we choose PP as a substrate because of the good trade-off between its thermal expansion coefficient $\left(a_{\mathrm{PP}} \approx 1.35 \cdot 10^{-4}{ }^{\circ} \mathrm{C}^{-1}\right)$ and its Young's modulus $\left(E_{P P} \approx 1.5 \mathrm{GPa}\right)$, which according to the finite element simulation give a strain transfer efficiency $g=0.75$ for a flake of $\mathrm{MoS}_{2}$.

We performed differential reflectance measurements as a function of substrate strain on single-layer flakes of $\mathrm{MoS}_{2}, \mathrm{MoSe}_{2}$, $\mathrm{WS}_{2}$ and $\mathrm{WSe}_{2}$ deposited on PP (see Supplementary Information Fig. SI13 for similar measurements performed on PDMS). Figure 4 displays the differential reflectance spectra of the four TMDCs single-layer flakes recorded at zero substrate expansion and at
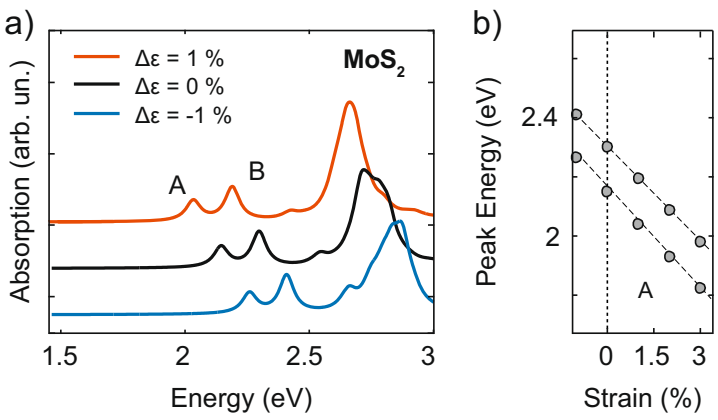

Fig. 3 Calculated BSE absorption spectra under biaxial strain of single-layer $\mathrm{MoS}_{2}$ (a). An artificial broadening of $0.035 \mathrm{eV}$ is used in the BSE calculations and the spectra are vertically shifted for improved visibility. The energies of the $A$ and $B$ excitons are extracted from the ab-initio calculations (b)
$0.9 \%$ of biaxial expansion, and at $0.1 \%$ of biaxial compression. All the spectra show maxima attributed to excitons in the materials ${ }^{36,37}$ on top of a broad background. An accurate theoretical description of the features present in the optical absorption spectra can be found in Ref. 41. At zero strain and energy lower than $2.2 \mathrm{eV}$, the Mo-based TMDCs show two peaks, already discussed in the case of $\mathrm{MoS}_{2}$, labelled A and B, whose separation is essentially equal to the spin-orbit splitting of the valence band. In the W-based TMDCs, the A peak is still evident, while the $B$ exciton is less prominent, giving rise to a shoulder in $\mathrm{WS}_{2}$ around $2.4 \mathrm{eV}$ and to a peak at $2.1 \mathrm{eV}$ in $\mathrm{WSe}_{2}$. The larger spin-orbit splitting, due to the heavier W atoms compared to Mo atoms, induces a larger separation of the $A$ and $B$ features in the differential reflectance spectra of $\mathrm{W}$-based TMDCs. The pronounced broad peak at energies above $2.5 \mathrm{eV}$ present in $\mathrm{MoS}_{2}, \mathrm{MoSe}_{2}$ and $\mathrm{WS}_{2}$ (C exciton) has been interpreted as coming from nearly-degenerate exciton states, located in regions of the Brillouin zone where the valence and conduction bands are nested. The different and more complicate physical origin of the peak $C$ in respect to the $A$ and $B$ excitonic peaks makes a direct comparison of the behaviour under strain difficult.

The $A$ and $B$ features shift to lower energies when the substrate expands and to higher energies for substrate compression. We extract the position of the A and B features as a function of substrate temperature for the four TMDCs and plot the results in Fig. 4. A linear fit to the data allows determining the gauge factors that are listed in Table 2 for each material. The magnitude of the shifts induced by the application of strain follow the order $\mathrm{WS}_{2}>$ $\mathrm{WSe}_{2}>\mathrm{MoS}_{2}>\mathrm{MoSe}_{2}$. Thus, given the same chalcogenide atom (S or Se), W atoms induce a larger gauge factor than Mo atoms, due to their more diffuse $d$ orbitals. Conversely, given the same metal, the lighter $\mathrm{S}$ atoms induce a larger shift than Se atoms. These effects and the trend observed are well reproduced by the calculations reported in Fig. 3 and in Supplementary Information Fig. SI20.

\section{DISCUSSION}

Our theoretically obtained gauge factors are systematically higher than our experimental values. In the experiment, as already discussed, only a part of the strain is transferred from the substrate to the monolayer. Therefore, the calculated values are an upper bound for the experimental gauge factors. The ordering of the gauge factors on the other hand for the different materials $\left(\mathrm{WS}_{2}>\right.$ $\mathrm{WSe}_{2}>\mathrm{MoS}_{2}>\mathrm{MoSe}_{2}$ ) is perfectly reproduced by our calculations. The magnitudes and ordering of the theoretical exciton gauge factors closely follow the gauge factors of the quasiparticle gap, as can be seen in Table 2, demonstrating that the underlying electronic structure already dictates the ordering. We find that the shift in the gaps under strain mainly stems from the shift of the conduction band minima (CBM). In the CBM, the shifts (with respect to the vacuum energies) are three times as high as in the valence band minima (VBM) for all four TMDCs. The magnitude of

Table 2. Comparison of the gauge factors of the four single-layer TMDCs deposited on PP

\begin{tabular}{|c|c|c|c|c|}
\hline Exciton & $\mathrm{MoS}_{2} / \mathrm{PP}$ & $\mathrm{MoSe}_{2} / \mathrm{PP}$ & $\mathrm{WS}_{2} / \mathrm{PP}$ & $\mathrm{WSe}_{2} / \mathrm{PP}$ \\
\hline B (experiment) & $-49 \mathrm{meV} / \%$ & $-30 \mathrm{meV} / \%$ & & $-43 \mathrm{meV} / \%$ \\
\hline B (theory) & $-107 \mathrm{meV} / \%$ & $-89 \mathrm{meV} / \%$ & $-130 \mathrm{meV} / \%$ & $-111 \mathrm{meV} / \%$ \\
\hline Quasiparticle band gap (theory) & $-134 \mathrm{meV} / \%$ & $-115 \mathrm{meV} / \%$ & $-156 \mathrm{meV} / \%$ & $-141 \mathrm{meV} / \%$ \\
\hline
\end{tabular}

Gauge factor for excitons A and B; the experimental values are extracted from applying biaxial strain on the different 2D flakes. The theoretical values stem from the calculated absorption spectra (BSE). Also included are the gauge factors of the quasiparticle gap, i.e. the VBM to CBM direct band gap at the K point 

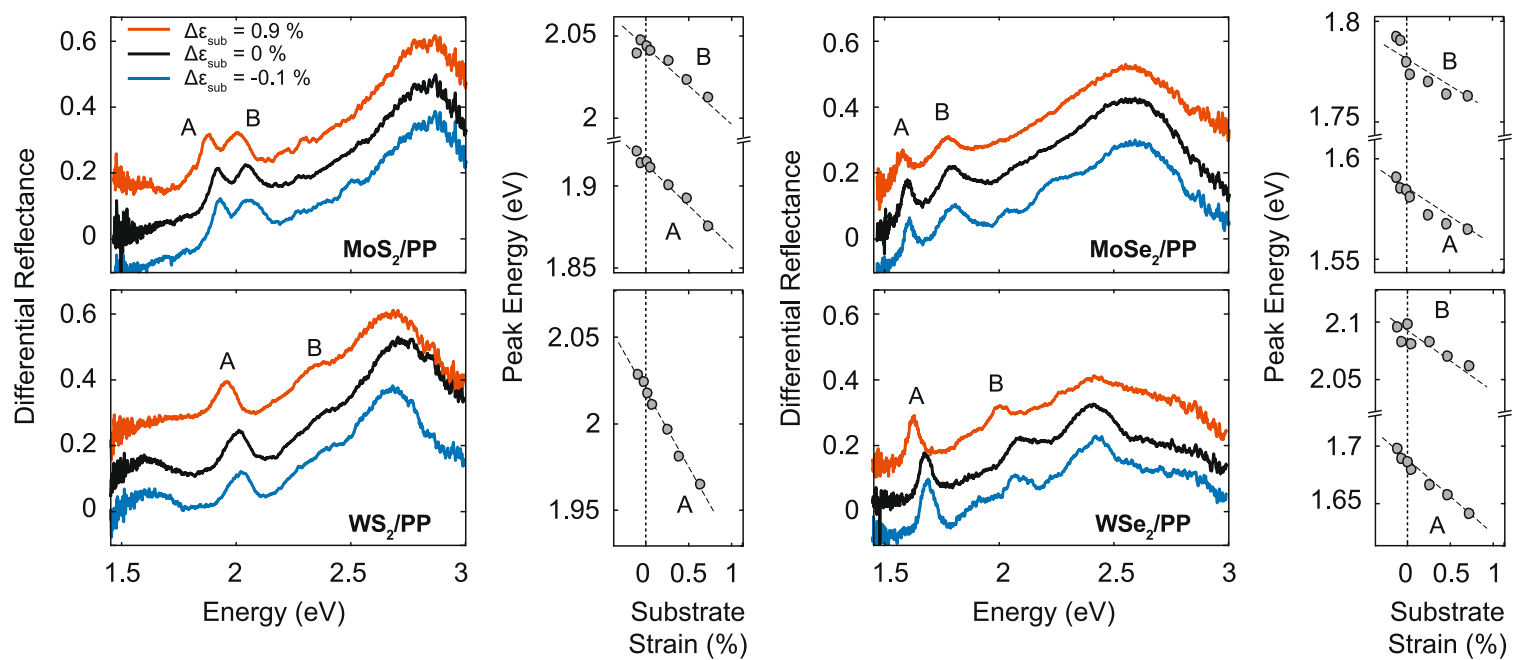

Fig. 4 Differential reflectance spectra of single-layer flakes of the four TMDCs deposited on PP. The spectra have been measured as a function of the substrate expansion (red curves) and contraction (black curves) and are vertically shifted for clarity. Energy of the excitonic peaks (labelled $A$ and $B$ ) as a function of substrate strain extracted from the differential reflectance spectra

the gauge factor ultimately measures how strong the electronic structure of the monolayer reacts to structural change. Strongly overlapping wave functions between atoms would lead to a larger gauge factor. The band width in the conduction band can be taken as a good indicator for the magnitude of the inter-atomic orbital overlap. Comparing the band width of the lowest conduction band around the $\mathrm{K}$ point (within a distance of $0.2 \pi / \mathrm{a}$ from $K$, the area of momentum space, which is mainly responsible for the $A$ and $B$ excitons), we find the ordering $W_{2}(0.30 \mathrm{eV})>$ $W_{S_{2}}(0.26 \mathrm{eV})>\mathrm{MoS}_{2}(0.20 \mathrm{eV})>\mathrm{MoSe}_{2}(0.18 \mathrm{eV})$ that follows the same ordering of the exciton gauge factors discussed previously. In conclusion, this suggests that the magnitude of the gauge factor, which mainly stems from the change in the CBM with strain, is largest for a strong overlap between atoms, which can be estimated through the band width around the $\mathrm{K}$ point. We notice that for the values of biaxial strain investigated, the four single-layer TMDCs remain direct gap semiconductors. ${ }^{4}, 13,18,39,42$

The strain-induced shift of the excitonic peaks in the differential reflection spectra of single-layer TMDCs is particularly interesting for applications as optical modulators. By controlling the temperature of the polymeric substrate, we can easily tune the position of the excitonic peaks and achieve a $10 \%$ modulation of the reflection for certain wavelengths in a time-scale of a few seconds. We record the differential reflectance of a $\mathrm{MoS}_{2}$ singlelayer flakes deposited on PP while cycling the temperature between 30 and $75^{\circ} \mathrm{C}$, applying a square wave modulated voltage to the Peltier heater. Figure $5 \mathrm{a}$ displays the differential reflectance at an energy of $1.91 \mathrm{eV}$, corresponding to a wavelength of $648 \mathrm{~nm}$, recorded as a function of time with a resolution of $300 \mathrm{~ms}$. The reflectance has a distorted square wave profile with rise/fall characteristic times of $10 \mathrm{~s}$ with a modulation of the intensity of $8 \%$ at $648 \mathrm{~nm}$. This timescale is limited by the heat transfer between the Peltier heater and the polymeric substrate, and could be improved by using local micro-heaters. Figure $5 \mathrm{~b}$ displays the position of the $A$ exciton peak as a function of time. The reproducibility in the shift and the transfer rate of the strain are excellent. The observed modulation in the signal of the order of $10 \%$ (at specific wavelengths) is noteworthy especially considering the atomic thickness $(<1 \mathrm{~nm})$ of these single-layer TMDCs. Modulations up to $25 \%$ can be reached thanks to the excitons that dominate the dielectric function of single-layer TMDCs materials, which are present at room temperature.
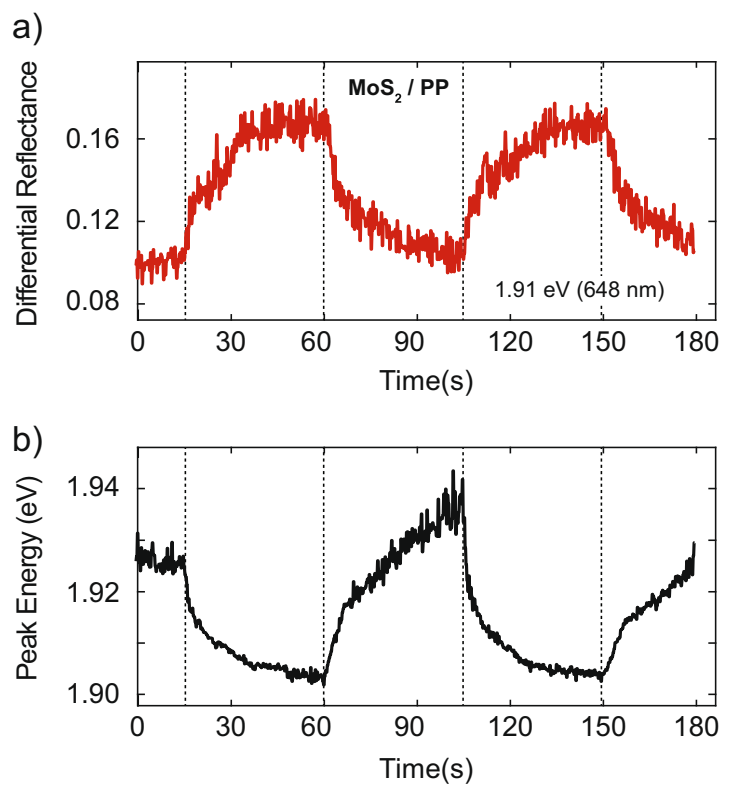

Fig. 5 a Differential reflectance measured at a wavelength of 648 $\mathrm{nm}$ on single-layer $\mathrm{MoS}_{2}$ deposited on PP as a function of time with periodical heating and cooling cycles of the substrates from 30 to $75^{\circ} \mathrm{C}$. Each differential reflectance spectrum has been integrated for $300 \mathrm{~ms}$. b Energy of exciton A as a function of time

In conclusion, we have exploited the large thermal expansion coefficient of a polymer substrate to apply large biaxial tensile strain on single-layer flakes of four TMDCs. By recording the exciton-dominated differential reflectance spectra of these materials as a function of external strain, we monitored the change in bandgap induced by the strain. We observe that the magnitude of the induced energy shift is the largest with $\mathrm{WS}_{2}$ flakes where it reaches $95 \mathrm{meV} / \%$. The shift follows the order $\mathrm{MoSe}_{2}<\mathrm{MoS}_{2}<\mathrm{WSe}_{2}<\mathrm{WS}_{2}$. Theoretical calculations reproduce well the experimental results and the observed trend in the various materials for the dependence of the bandgap on the 
external strain. The large shift induced in the bandgap by biaxial strain and the rapidity for the transfer of strain open the possibility for the use of $2 \mathrm{D}$ TMDCs as electro-optical modulators or strain sensors.

\section{METHODS}

\section{Differential reflectance measurements}

White light from a halogen lamp irradiates the sample after passing through a small diaphragm and results in an illumination spot of approximately $50 \mu \mathrm{m}$ at the sample surface. The light reflected from the sample is collected with an optical fibre (105 $\mu \mathrm{m}$ core diameter) and fed to a spectrometer. The fibre is used to collect only the light reflected from a few $\mu \mathrm{m}$ area of the sample located approximately in the center of the illumination spot. The differential reflectance spectrum of a TMDC flake is calculated by subtracting from the reflectance spectrum collected on top of the flake the same spectrum collected on the substrate, and normalising the result by the flake spectrum.

\section{Ab-initio calculations}

We performed ab-initio DFT calculation in the LDA, from which we obtain the energetically optimised structure. The DFT wave functions and energies are then used as input for a subsequent GW calculation within the LDA+GdW approximation, ${ }^{36}$ in which the dielectric screening properties are described by an atom-resolved model function based on the random-phase approximation. ${ }^{36}$ In the last step, the BSE is set up from the quasiparticle band structure. For the given TMDC monolayers, excitons are composed from four valence and six conduction bands and a mesh of $30 \times 30 \times 1 \mathrm{k}$ points from the first Brillouine zone. To simulate biaxial tensile (compressive) strain, the lattice constant is increased (decreased) fully relaxing the structure for each applied strain. All further numerical details can be found in the Supplementary Information. This approach has already been successfully used to describe uniaxial strain in a $\mathrm{WS}_{2}$ monolayer. ${ }^{19}$

\section{ACKNOWLEDGEMENTS}

We thank Dr. Isabel Rodriguez for the nanoimprinted PDMS substrates and Dr. Emilio Pérez Alvarez and Alejandro López Moreno for their help with the Raman measurements. We acknowledge financial support from the European Commission, the MINECO, the Comunidad de Madrid and from the Netherlands Organisation for Scientific Research (NWO). M.D. thanks the Studienstiftung des deutschen Volkes for support via a PhD fellowship. The authors gratefully acknowledge the computing time granted by the John von Neumann Institute for Computing (NIC) and provided on the supercomputer JURECA at Jülich Supercomputing Centre (JSC). A.C.G. European Commission under the Graphene Flagship: contract CNECTICT-604391 A.C. G. MINECO: Ramón y Cajal 2014 program RYC-2014- 01406 A.C.GG. MINECO: progeam MAT2014-58399-JIN A.C.G. Comunidad de Madrid: MAD2D-CM program (S2013/MIT3007) R.F. Netherlands Organisation for Scientific Research (NWO): Rubicon 680-501515 D.P.dL. MINECO: program FIS2015-67367-C2-1-p.

\section{AUTHOR CONTRIBUTIONS}

R.F. and A.C.G. carried out the strain experiments. R.F analysed the experimental data. M.D and M.R. performed the ab-initio calculations. A.C.G. and D.PdL. designed the experiment. R.S., S.M.dV. and R.B. performed the finite element simulations. All the authors discussed the results and contributed to the writing of the manuscript.

\section{COMPETING INTERESTS}

The authors declare that they have no competing financial interests.

\section{REFERENCES}

1. Roldan, R., Castellanos-Gomez, A., Cappelluti, E. \& Guinea, F. Strain engineering in semiconducting two-dimensional crystals. J. Phys. Condens. Matter 27, 313201 (2015).

2. Bissett, M. A., Tsuji, M. \& Ago, H. Strain engineering the properties of graphene and other two-dimensional crystals. Phys. Chem. Chem. Phys. 16, 11124-11138 (2014).

3. Zhang, G. \& Zhang, Y.-W. Strain effects on thermoelectric properties of twodimensional materials. Mech. Mater. 91, 382-398 (2015).
4. Horzum, S. et al. Phonon softening and direct to indirect band gap crossover in strained single-layer MoSe ${ }_{2}$. Phys. Rev. B 87, 125415 (2013).

5. Bertolazzi, S., Brivio, J. \& Kis, A. Stretching and breaking of ultrathin $\mathrm{MoS}_{2}$. ACS Nano 5, 9703-9709 (2011).

6. Coleman, J. N. et al. Two-dimensional nanosheets produced by liquid exfoliation of layered materials. Science 331, 568-571 (2011).

7. Clarke, D. R. in Semiconductors and Semimetals, Vol. 37 (eds Faber Katherine, T. \& Malloy Kevin, J.) 79-142 (Elsevier, 1992).

8. Crowne, F. J. et al. Blueshift of the A-exciton peak in folded monolayer $1 \mathrm{H}-\mathrm{MoS}_{2}$. Phys. Rev. B 88, 235302 (2013).

9. Hui, Y. Y. et al. Exceptional tunability of band energy in a compressively strained trilayer $\mathrm{MoS}_{2}$ sheet. ACS Nano 7, 7126-7131 (2013).

10. Wang, Y., Cong, C., Qiu, C. \& Yu, T. Raman spectroscopy study of lattice vibration and crystallographic orientation of monolayer $\mathrm{MoS}_{2}$ under uniaxial strain. Small $\mathbf{9}$, 2857-2861 (2013).

11. Liu, Z. et al. Strain and structure heterogeneity in $\mathrm{MoS}_{2}$ atomic layers grown by chemical vapour deposition. Nat. Commun. 5, 5246 (2014).

12. Plechinger, G. et al Control of biaxial strain in single-layer molybdenite using local thermal expansion of the substrate. 2D Mater. 2, 015006 (2015).

13. Wang, Y. et al. Strain-induced direct-indirect bandgap transition and phonon modulation in monolayer $\mathrm{WS}_{2}$. Nano Res 8, 2562-2572 (2015).

14. Lloyd, D. et al. Band gap engineering with ultralarge biaxial strains in suspended monolayer $\mathrm{MoS}_{2}$. Nano Lett. 16, 5836-5841 (2016).

15. Conley, H. J. et al. Bandgap engineering of strained monolayer and bilayer $\mathrm{MoS}_{2}$. Nano Lett. 13, 3626-3630 (2013).

16. He, K., Poole, C., Mak, K. F. \& Shan, J. Experimental demonstration of continuous electronic structure tuning via strain in atomically thin $\mathrm{MoS}_{2}$. Nano Lett. 13, 2931-2936 (2013).

17. Zhu, C. R. et al. Strain tuning of optical emission energy and polarization in monolayer and bilayer $\mathrm{MoS}_{2}$. Phys. Rev. B 88, 121301 (2013).

18. Desai, S. B. et al. Strain-induced indirect to direct bandgap transition in multilayer WSe $e_{2}$. Nano Lett. 14, 4592-4597 (2014).

19. Island, J. O. et al. Precise and reversible band gap tuning in single-layer $\mathrm{MoSe}_{2}$ by uniaxial strain. Nanoscale 8, 2589-2593 (2016).

20. Robert, S. et al. Reversible uniaxial strain tuning in atomically thin $\mathrm{WSe}_{2} .2 D$ Mater. 3, 021011 (2016).

21. McCreary, A. et al. Effects of uniaxial and biaxial strain on few-layered terrace structures of $\mathrm{MoS}_{2}$ grown by vapor transport. ACS Nano 10, 3186-3197 (2016).

22. Castellanos-Gomez, A. et al. Local strain engineering in atomically thin $\mathrm{MoS}_{2}$. Nano Lett. 13, 5361-5366 (2013).

23. Quereda, J. et al. Strong modulation of optical properties in Black Phosphorus through strain-engineered rippling. Nano Lett. 16, 2931-2937 (2016).

24. Yang, S. et al. Tuning the optical, magnetic, and electrical properties of $\mathrm{ReSe}_{2}$ by nanoscale strain engineering. Nano Lett. 15, 1660-1666 (2015).

25. Manzeli, S., Allain, A., Ghadimi, A. \& Kis, A. Piezoresistivity and strain-induced band gap tuning in atomically thin $\mathrm{MoS}_{2}$. Nano Lett. 15, 5330-5335 (2015).

26. Tongay, S. et al. Monolayer behaviour in bulk $\mathrm{ReS}_{2}$ due to electronic and vibrational decoupling. Nat. Commun. 5, 3252 (2014).

27. Gong, L. et al. Interfacial stress transfer in a graphene monolayer nanocomposite. Adv. Mater. 22, 2694-2697 (2010).

28. Mark, J. E. Polymer data handbook (Oxford university press, 2009).

29. Castellanos-Gomez, A. et al. Elastic properties of freely suspended $\mathrm{MoS}_{2}$ nanosheets. Adv. Mater. 24, 772-775 (2012).

30. Castellanos-Gomez, A. et al. Deterministic transfer of two-dimensional materials by all-dry viscoelastic stamping. 2D Mater. 1, 011002 (2014).

31. Lee, C. et al. Anomalous lattice vibrations of single- and few-layer $\mathrm{MoS}_{2}$. ACS Nano 4, 2695-2700 (2010)

32. Molina-Sánchez, A. \& Wirtz, L. Phonons in single-layer and few-layer $\operatorname{MoS}_{2}$ and WS $_{2}$. Phys. Rev. B 84, 155413 (2011).

33. Dhakal, K. P. et al. Confocal absorption spectral imaging of $\mathrm{MoS}_{2}$ : optical transitions depending on the atomic thickness of intrinsic and chemically doped $\mathrm{MoS}_{2}$. Nanoscale 6, 13028-13035 (2014).

34. Mclntyre, J. \& Aspnes, D. Differential reflection spectroscopy of very thin surface films. Surf. Sci. 24, 417-434 (1971).

35. Ross, J. S. et al. Electrical control of neutral and charged excitons in a monolayer semiconductor. Nat. Commun. 4, 1474 (2013).

36. Kozawa, D. et al. Photocarrier relaxation pathway in two-dimensional semiconducting transition metal dichalcogenides. Nat. Commun. 5, 4543 (2014).

37. $\mathrm{Yu}, \mathrm{Y}$. et al. Exciton-dominated dielectric function of atomically thin $\mathrm{MoS}_{2}$ films. Sci. Rep. 5, 16996 (2015).

38. Li, Y. et al. Measurement of the optical dielectric function of monolayer transitionmetal dichalcogenides: $\mathrm{MoS}_{2}, \mathrm{MoSe}_{2}, \mathrm{WS}_{2}$, and WSe 2 . Phys. Rev. B 90, 205422 (2014).

39. Tongay, S. et al. Thermally driven crossover from indirect toward direct bandgap in 2D semiconductors: $\mathrm{MoSe}_{2}$ versus $\mathrm{MoS}_{2}$. Nano Lett. 12, 5576-5580 (2012). 
40. Rohlfing, M. Electronic excitations from a perturbative LDA+GdW approach. Phys. Rev. B 82, 205127 (2010).

41. Gillen, R. \& Maultzsch, J. Light-matter interactions in two-dimensional transition metal dichalcogenides: dominant excitonic transitions in mono- and few-layer $\mathrm{MoX}_{2}$ and band nesting. IEEE J. Sel. Top. Quantum Electron. 23, 1-12 (2017).

42. Sahin, $H$. et al. Anomalous Raman spectra and thickness-dependent electronic properties of $\mathrm{WSe}_{2}$. Phys. Rev. B 87, 165409 (2013).

Open Access This article is licensed under a Creative Commons Attribution 4.0 International License, which permits use, sharing, adaptation, distribution and reproduction in any medium or format, as long as you give appropriate credit to the original author(s) and the source, provide a link to the Creative Commons license, and indicate if changes were made. The images or other third party material in this article are included in the article's Creative Commons license, unless indicated otherwise in a credit line to the material. If material is not included in the article's Creative Commons license and your intended use is not permitted by statutory regulation or exceeds the permitted use, you will need to obtain permission directly from the copyright holder. To view a copy of this license, visit http://creativecommons. org/licenses/by/4.0/.

(c) The Author(s) 2017

Supplementary Information accompanies the paper on the npj 2D Materials and Applications website (doi:10.1038/s41699-017-0013-7). 